\title{
PACIENTE COM PARALISA CEREBRAL COREOATETOIDE: EVOLUÇÃO CLÍNICA PÓS-INTERVENÇÃO
}

\section{Patient with choreoathetoid cerebral palsy: post therapeutic intervention}

\author{
Carolina Castelli Silvério ${ }^{(1)}$, Cristiane Soares Henrique ${ }^{(2)}$
}

\begin{abstract}
RESUMO
Objetivo: verificar a eficácia da intervenção terapêutica na função de deglutição e na estabilidade clínica de crianças portadoras de paralisia cerebral (PC) coreoatetoide com disfagia orofaríngea. Métodos: 11 crianças portadoras de PC do tipo coreoatetoide, com média de idade de três anos e três meses, que frequentaram terapia fonoaudiológica. Foram levantados os seguintes dados: escala de avaliação funcional da alimentação (Functional Oral Intake Scale - FOIS); grau de severidade da disfagia; broncopneumonias (BCP), hipersecretividade pulmonar e peso; consistência alimentar; sinais de penetração e/ou aspiração laringotraqueal. Os dados foram levantados no relatório de avaliação antes da intervenção terapêutica e no relatório final de evolução, no momento da alta. Resultados: com relação à aplicação da escala FOIS, obteve-se que, tanto antes, quanto após a intervenção, a maioria dos pacientes encontrou-se no nível $V$ da escala, com diminuição no segundo momento. Aumento dos pacientes nos níveis III e IV. Após intervenção terapêutica, houve diminuição de pacientes que ingeriam líquidos, aumento do uso do líquido espesso e do pastoso homogêneo. Houve diminuição da severidade da disfagia, redução dos episódios de BCP e de hipersecretividade pulmonar, aumento de peso e redução dos sinais de penetração e/ou aspiração laringotraqueal. Conclusão: a intervenção fonoaudiológica, dentro de uma equipe multidisciplinar em disfagia, em crianças portadoras de PC coreoatetoide promove deglutição mais segura e eficaz, com redução dos sinais sugestivos de penetração e/ou aspiração laringotraqueal, dos episódios de BCP e de hipersecretividade pulmonar, e aumento do peso.
\end{abstract}

DESCRITORES: Paralisia Cerebral; Transtornos de Deglutição; Fonoterapia

\section{INTRODUÇÃO}

A disfagia orofaríngea compreende uma alteração que afeta o transporte do bolo alimentar da boca até o estômago, comprometendo a eficácia e segurança do processo da deglutição, e encontra-se frequentemente presente em crianças portadoras de paralisia cerebral. Sendo verificada tal dificuldade, essas crianças são encaminhadas para terapia fonoaudiológica, visando otimizar o processo da deglutição, diminuindo seus riscos. A presença da disfagia orofaríngea pode trazer complicações como intercorrências respiratórias, desnutrição e desidratação ${ }^{1,2}$.

(1) Fonoaudióloga Responsável pela Clínica de Disfagia da Associação de Assistência à Criança Deficiente, AACD, São Paulo, SP; Mestre em Ciências pela Universidade Federal de São Paulo.

(2) Fonoaudióloga do Hospital Sírio Libanês, São Paulo, SP. Conflito de interesses: inexistente
A paralisia cerebral coreoatetoide compreende uma alteração neurológica central, não evolutiva, que compromete o movimento e a postura, sendo caracterizada principalmente pela presença de movimentos involuntários. A criança com paralisia cerebral coreoatetoide pode apresentar movimentação involuntária de língua e de mandíbula, interferindo na dinâmica da deglutição ${ }^{3}$.

Verifica-se a necessidade de mensurar a eficácia da intervenção fonoaudiológica dentro de uma equipe multidisciplinar em disfagia orofaríngea, nas crianças com paralisia cerebral, uma vez que existem poucos estudos na literatura que comprovem esse fato ${ }^{4}$. Sabe-se que esta atuação frente aos diferentes tipos de paralisia cerebral, seja através de técnicas terapêuticas, ou de orientações aos cuidadores, apresenta o mesmo embasamento. Porém, em virtude da diferença do comprometimento motor global e do melhor desempenho cognitivo geralmente observado nas crianças com 
paralisia cerebral coreoatetoide, torna-se necessário um estudo somente com este grupo de crianças.

Assim, este estudo tem como objetivo verificar a eficácia da intervenção terapêutica na função de deglutição e na estabilidade clínica de crianças portadoras de paralisia cerebral coreoatetoide com disfagia orofaríngea.

\section{MÉTODOS}

Participaram do estudo 11 crianças portadoras de paralisia cerebral do tipo coreoatetoide, sendo oito do sexo masculino e três do feminino, com média de idade de três anos e três meses, que frequentaram terapia fonoaudiológica semanal de 40 minutos, por uma média de 9 meses, com no mínimo de 3 meses de atendimento, com objetivos relacionados à disfagia orofaríngea, no Ambulatório de Fonoaudiologia da Associação de Assistência à Criança Deficiente de São Paulo, SP.

Foram consultados os prontuários dos pacientes que passaram em terapia no setor de Fonoaudiologia/Disfagia.

Para o levantamento dos dados foi criado um Protocolo de Evolução, que consta de:

- Functional Oral Intake Scale ${ }^{5}$ (FOIS), que compreende uma escala de avaliação funcional da alimentação, permitindo classificar o paciente conforme a via de alimentação utilizada. Os níveis são:

- Nível I: alimentação por via alternativa, sem oferta por via oral;

- Nível II: alimentação por via alternativa, com estimulação gustativa por via oral;

- Nível III: alimentação por via alternativa, com oferta constante de alimento por via oral;

- Nível IV: alimentação por via oral, de única consistência, sendo que as consistências pastoso homogêneo e líquido espesso são consideradas pela escala como consistência única;

- Nível V: alimentação por via oral, com múltiplas consistências, mas que necessitam de preparo especial;

- Nível VI: alimentação por via oral, com múltiplas consistências sem preparo especial, porém com limitações específicas, como por exemplo, evitar grãos secos, ou líquidos com gás;

- Nível VII: alimentação por via oral sem restrições.

- Classificação do grau de severidade da Disfagia Orofaríngea que pode ser Leve, Moderada e Grave, de acordo com o Protocolo ROGS 6;

- Presença de broncopneumonias (BCP), hipersecretividade pulmonar e ganho de peso;

- Consistências alimentares ingeridas: líquido, líquido espesso, pastoso homogêneo, pastoso heterogêneo e sólido.
Presença de sinais sugestivos de penetração e/ ou aspiração laringotraqueal, como tosse, engasgo, desconforto alimentar, ausculta cervical ruidosa, deglutições múltiplas e fadiga.

Estes dados foram colhidos no relatório de Avaliação Fonoaudiológica de Disfagia, que é realizado no início da terapia, e nos relatórios de Evolução que são preenchidos a cada três meses no período de terapia.

Durante a intervenção foram realizadas orientações quanto à postura, utensílios, consistência e volume alimentar mais adequado para cada caso, manuseios motores globais (Conceito Bobath ${ }^{7}$ ) que visam adequar tônus e postura para benefício da função de alimentação, estimulações extra e intraorais e terapia de alimentação, na qual, utilizandose da oferta de alimento, busca-se melhor funcionalidade da deglutição. Além disso, houve a atuação da equipe multidisciplinar de disfagia, composta por médicos fisiatra, gastroenterologista, otorrinolaringologista, e pneumologista, fonoaudiólogo, fisioterapeuta respiratório e nutricionista.

Esta pesquisa foi aprovada pelo Comitê de Ética em Pesquisa da Associação de Assistência à Criança Deficiente, protocolo número 10/2006.

$\mathrm{Na}$ análise dos resultados foi aplicado o Teste dos Postos Sinalizados de Wilcoxon a fim de estudar possíveis diferenças entre os momentos pré e pós-intervenção. Fixou-se em 0,05 ou 5\% o nível de rejeição da hipótese de nulidade.

\section{RESULTADOS}

Com relação à aplicação da escala FOIS, obteve-se como resultado (Tabela $1-p=0,408$ ) que tanto antes da intervenção $(81,82 \%)$, quanto após esta $(45,45 \%)$, a maioria dos pacientes encon-

Tabela 1 - Distribuição numérica e percentual dos pacientes por nível da escala FOIS nos momentos pré e pós-intervenção $(p=0,408)$

\begin{tabular}{ccc}
\hline \multirow{2}{*}{ Nível FOIS } & \multicolumn{2}{c}{ Fonoterapia } \\
\cline { 2 - 3 } & Pré & Pós \\
& $\mathbf{N}(\%)$ & $\mathbf{N}(\%)$ \\
\hline I & $1(9,09)$ & $0(0)$ \\
II & $0(0)$ & $0(0)$ \\
III & $0(0 \%)$ & $2(18,18 \%)$ \\
IV & $0(0)$ & $3(27,27 \%)$ \\
V & $9(81,82 \%)$ & $5(45,45 \%)$ \\
VI & $1(9,09 \%)$ & $1(9,09 \%)$ \\
VII & $0(0)$ & $0(0)$ \\
Total & $11(100 \%)$ & $11(100 \%)$ \\
\hline
\end{tabular}

Legenda: $p=0,408 ; N=$ número de pacientes 
Tabela 2 - Distribuição numérica e percentual dos pacientes de acordo com a consistência alimentar ingerida, nos momentos pré e pós-intervenção

\begin{tabular}{|c|c|c|c|c|c|c|c|c|c|c|}
\hline \multirow{2}{*}{$\begin{array}{l}\text { Consistências } \\
\text { Alimentares }\end{array}$} & \multicolumn{2}{|c|}{$\begin{array}{l}\text { Líquido } \\
\left(p<0,025^{\star}\right)\end{array}$} & \multicolumn{2}{|c|}{$\begin{array}{c}\text { Líquido } \\
\text { espesso } \\
\left(p=0,046^{*}\right)\end{array}$} & \multicolumn{2}{|c|}{$\begin{array}{c}\text { Pastoso } \\
\text { homogêneo } \\
\left(p=0,046^{*}\right)\end{array}$} & \multicolumn{2}{|c|}{$\begin{array}{c}\text { Pastoso } \\
\text { heterogêneo } \\
\left(p=0,046^{*}\right)\end{array}$} & \multicolumn{2}{|c|}{$\begin{array}{c}\text { Sólido } \\
(p<0,999)\end{array}$} \\
\hline & $\begin{array}{l}\text { Pré } \\
\text { N (\%) }\end{array}$ & $\begin{array}{l}\text { Pós } \\
\text { N (\%) }\end{array}$ & $\begin{array}{l}\text { Pré } \\
\text { N (\%) }\end{array}$ & $\begin{array}{l}\text { Pós } \\
\text { N (\%) }\end{array}$ & $\begin{array}{c}\text { Pré } \\
\text { N (\%) }\end{array}$ & $\begin{array}{l}\text { Pós } \\
\text { N (\%) }\end{array}$ & $\begin{array}{c}\text { Pré } \\
\text { N (\%) }\end{array}$ & $\begin{array}{l}\text { Pós } \\
\text { N (\%) }\end{array}$ & $\begin{array}{c}\text { Pré } \\
\text { N (\%) }\end{array}$ & $\begin{array}{l}\text { Pós } \\
\text { N (\%) }\end{array}$ \\
\hline Ingerem & $\begin{array}{c}10 \\
(100 \%)\end{array}$ & $\begin{array}{c}5 \\
(50 \%)\end{array}$ & $\begin{array}{c}0 \\
(0 \%)\end{array}$ & $\begin{array}{c}4 \\
(40 \%)\end{array}$ & $\begin{array}{c}5 \\
(50 \%)\end{array}$ & $\begin{array}{c}9 \\
(90 \%)\end{array}$ & $\begin{array}{c}9 \\
(90 \%)\end{array}$ & $\begin{array}{c}5 \\
(50 \%)\end{array}$ & $\begin{array}{c}0 \\
(0 \%)\end{array}$ & $\begin{array}{c}0 \\
(0 \%)\end{array}$ \\
\hline Não ingerem & $\begin{array}{c}0 \\
(0 \%)\end{array}$ & $\begin{array}{c}5 \\
(50 \%)\end{array}$ & $\begin{array}{c}10 \\
(76,67 \%)\end{array}$ & $\begin{array}{c}6 \\
(60 \%)\end{array}$ & $\begin{array}{c}5 \\
(50 \%)\end{array}$ & $\begin{array}{c}1 \\
(10 \%)\end{array}$ & $\begin{array}{c}1 \\
(10 \%)\end{array}$ & $\begin{array}{c}5 \\
(50 \%)\end{array}$ & $\begin{array}{c}0 \\
(0 \%)\end{array}$ & $\begin{array}{c}0 \\
(0 \%)\end{array}$ \\
\hline Total & $\begin{array}{c}10 \\
(100 \%)\end{array}$ & $\begin{array}{c}10 \\
(100 \%)\end{array}$ & $\begin{array}{c}10 \\
(100 \%)\end{array}$ & $\begin{array}{c}10 \\
(100 \%)\end{array}$ & $\begin{array}{c}10 \\
(100 \%)\end{array}$ & $\begin{array}{c}10 \\
(100 \%)\end{array}$ & $\begin{array}{c}10 \\
(100 \%)\end{array}$ & $\begin{array}{c}10 \\
(100 \%)\end{array}$ & $\begin{array}{c}0 \\
(0 \%)\end{array}$ & $\begin{array}{c}0 \\
(0 \%)\end{array}$ \\
\hline
\end{tabular}

Legenda: $\mathrm{N}=$ número de pacientes

trou-se no Nível $V$ da escala, com diminuição no segundo momento. Foi possível verificar aumento dos pacientes nos Níveis III e IV, de 0 para 18,18\% e de 0 para $27,27 \%$ respectivamente.

O resultado das consistências alimentares, conforme pode ser observado na Tabela 2, demonstra que após intervenção terapêutica houve diminuição da porcentagem de pacientes que ingeriam líquidos (de $100 \%$ para $50 \%$ - $p=0,025^{\star}$ ), aumento do uso do líquido espesso (de nenhum para $40 \%$ $\mathrm{p}=0,046^{\star}$ ) e do pastoso homogêneo (de $50 \%$ para $\left.90 \%-p=0,046^{\star}\right)$, e diminuição da ingesta do pastoso heterogêneo (de $90 \%$ para $50 \%-p=0,046^{*}$ ). No que diz respeito à consistência alimentar sólida, observou-se ausência desta na alimentação dos pacientes da pesquisa, tanto no momento pré quanto no pós-intervenção.

No resultado referente ao grau de disfagia, verificou-se após a intervenção, diminuição dos pacientes com Disfagia Grave (de 36,36\% para $9,09 \%$ ) e aumento daqueles com Disfagia Leve (de $18,18 \%$ para $45,45 \%$ ). A porcentagem da presença de Disfagia Moderada manteve-se a mesma, sendo $45,45 \%$ dos pacientes, conforme pode ser observado na Tabela $3\left(p=0,034^{*}\right)$

$\mathrm{Na}$ Tabela 4, a análise dos episódios de BCP $\left(p=0,021^{*}\right)$ revelou que no momento pós-intervenção, houve aumento do número de pacientes sem episódios de BCP (de 36,36\% para 90,91\%). Na mesma tabela, também é possível observar o resultado relacionado com a presença de hipersecretividade pulmonar $\left(p=0,046^{*}\right)$, com diminuição desta de $63,64 \%$ para $27,27 \%$ dos pacientes no momento pós-intervenção terapêutica.

Dos 11 pacientes deste estudo, somente em sete deles foi possível realizar a análise do peso em decorrência de falta de preenchimento deste dado nas fichas de avaliação. Nestes sete pacientes foi possível verificar aumento da média do peso
Tabela 3 - Distribuição numérica e percentual dos pacientes de acordo com o grau de severidade da disfagia orofaríngea (protocolo ROGS) nos momentos pré e pós-intervenção $\left(p=0,034^{*}\right)$

\begin{tabular}{ccc}
\hline $\begin{array}{c}\text { Grau de } \\
\text { Severidade }\end{array}$ & $\begin{array}{c}\text { Pré-fonoterapia } \\
\text { N (\%) }\end{array}$ & $\begin{array}{c}\text { Pós- } \\
\text { fonoterapia } \\
\mathbf{N ~ ( \% )}\end{array}$ \\
\hline Leve & $2(18,18 \%)$ & $5(45,45 \%)$ \\
Moderado & $5(45,45 \%)$ & $5(45,45 \%)$ \\
Grave & $4(36,36 \%)$ & $1(9,09 \%)$ \\
Total & $11(100,00 \%)$ & $11(100,00 \%)$ \\
\hline
\end{tabular}

Legenda: $p=0,034^{*} ; N=$ número de pacientes

de $10,69 \pm 2,54$ no momento pré-intervenção, para $11,57 \pm 2,57$ no pós.

Na Tabela 5 verificou-se redução na incidência dos sinais sugestivos de penetração e/ou aspiração laringotraqueal, comparando os momentos pré e pós-intervenção. Os episódios de tosse e a presença de ausculta cervical positiva tiveram redução significante, de $81,82 \%$ para $27,27 \% \quad\left(p=0,014^{*}\right)$ para a tosse, e de $81,82 \%$ para $18,18 \%\left(p=0,008^{*}\right)$ para a ausculta cervical positiva.

\section{DISCUSSÃO}

A aplicação da escala FOIS e a análise das consistências alimentares ingeridas pelos pacientes permitem verificar que após a intervenção fonoaudiológica há maior homogeneidade entre as consistências nas crianças com PC coreoatetoide. O aumento do Nível IV da escala FOIS revela o aumento do uso dos líquidos espessados, tornando a alimentação em uma única consistência. Além 
Tabela 4 - Distribuição numérica e percentual dos pacientes por número de broncopneumonias $B C P\left(P=0,021^{\star}\right)$ e presença de hipersecretividade $\left(p=0,046^{\star}\right)$ nos momentos pré e pós-intervenção

\begin{tabular}{ccc}
\hline NÚMERO DE & $\begin{array}{c}\text { Pré-fonoterapia } \\
\text { N (\%) }\end{array}$ & $\begin{array}{c}\text { Pós-fonoterapia } \\
\text { N (\%) }\end{array}$ \\
\hline BRONCOPNEUMONIA & $4(36,36 \%)$ & $10(90,91 \%)$ \\
Nenhum & $2(18,18 \%)$ & $1(9,09 \%)$ \\
2 & $5(45,45 \%)$ & $0(0 \%)$ \\
5 ou mais & $11(100,00 \%)$ & $11(100,00 \%)$ \\
Total & & \\
HIPERSECRETIVIDADE & $7(63,64 \%)$ & $3(27,27 \%)$ \\
Presente & $4(36,36 \%)$ & $8(72,73 \%)$ \\
Ausente & $11(100,00 \%)$ & $11(100,00 \%)$ \\
Total & &
\end{tabular}

Legenda: $\mathrm{N}=$ número de pacientes

Tabela 5 - Distribuição numérica e percentual dos pacientes de acordo com a presença dos sinais sugestivos de penetração e/ou aspiração laringotraqueal, nos momentos pré e pós-intervenção

\begin{tabular}{|c|c|c|c|c|c|c|}
\hline \multirow[t]{2}{*}{ Sinal } & \multicolumn{2}{|c|}{$\begin{array}{c}\text { Tosse } \\
\left(p=0,014^{\star}\right)\end{array}$} & \multicolumn{2}{|c|}{$\begin{array}{l}\text { Engasgo } \\
(p=0,083)\end{array}$} & \multicolumn{2}{|c|}{$\begin{array}{c}\text { Desconforto } \\
(p=0,059)\end{array}$} \\
\hline & $\begin{array}{c}\text { Pré } \\
\text { N (\%) }\end{array}$ & $\begin{array}{l}\text { Pós } \\
\text { N (\%) }\end{array}$ & $\begin{array}{c}\text { Pré } \\
\text { N (\%) }\end{array}$ & $\begin{array}{l}\text { Pós } \\
\text { N (\%) }\end{array}$ & $\begin{array}{c}\text { Pré } \\
\text { N (\%) }\end{array}$ & $\begin{array}{l}\text { Pós } \\
\text { N (\%) }\end{array}$ \\
\hline Presente & $9(81,82 \%)$ & $3(27,27 \%)$ & $7(63,64 \%)$ & $4(36,36 \%)$ & $8(72,73 \%)$ & $3(27,27 \%)$ \\
\hline Ausente & $2(18,18 \%)$ & $8(72,73 \%)$ & $4(36,36 \%)$ & $7(63,64 \%)$ & $3(27,27 \%)$ & $8(72,73 \%)$ \\
\hline Total & $11(100 \%)$ & $11(100 \%)$ & $11(100 \%)$ & $11(100 \%)$ & $11(100 \%)$ & $11(100 \%)$ \\
\hline \multirow[t]{2}{*}{ Sinal } & \multicolumn{2}{|c|}{$\begin{array}{c}\text { Ausculta Cervical } \\
\text { Positiva } \\
\left(p=0,008^{*}\right)\end{array}$} & \multicolumn{2}{|c|}{$\begin{array}{l}\text { Deglutições Múltiplas } \\
\qquad(p=0,317)\end{array}$} & \multicolumn{2}{|c|}{$\begin{array}{c}\text { Fadiga } \\
(p=0,083)\end{array}$} \\
\hline & $\begin{array}{l}\text { Pré } \\
\text { N (\%) }\end{array}$ & $\begin{array}{l}\text { Pós } \\
\text { N (\%) }\end{array}$ & $\begin{array}{l}\text { Pré } \\
\text { N (\%) }\end{array}$ & $\begin{array}{l}\text { Pós } \\
\text { N (\%) }\end{array}$ & $\begin{array}{l}\text { Pré } \\
\text { N (\%) }\end{array}$ & $\begin{array}{l}\text { Pós } \\
\text { N (\%) }\end{array}$ \\
\hline Presente & $9(81,82 \%)$ & $2(18,18 \%)$ & $6(54,55 \%)$ & $4(36,36 \%)$ & $4(36,36 \%)$ & $1(9,09 \%)$ \\
\hline Ausente & $2(18,18 \%)$ & $9(81,82 \%)$ & $5(45,45 \%)$ & $7(63,64 \%)$ & $7(63,64 \%)$ & $10(90,91 \%)$ \\
\hline Total & $11(100 \%)$ & $11(100 \%)$ & $11(100 \%)$ & $11(100 \%)$ & $11(100 \%)$ & $11(100 \%)$ \\
\hline
\end{tabular}

disso, pode-se observar redução do consumo do líquido fino, confirmando tal dado. Pesquisas 8-10 afirmam que a consistência alimentar mais frequentemente aspirada é a líquida. Furkim e Sacco ${ }^{11}$ verificam que o padrão de coordenação deglutição e respiração diferencia-se nas deglutições de líquidos finos ou de pastoso em pacientes com PC, com a maioria destes demonstrando inspiração após a deglutição depois da deglutição do líquido fino. Os autores afirmam que tal achado possa ser decorrente do pobre controle oral do bolo. Outro estudo também refere progressão da funcionalidade da deglutição após fonoterapia, usando como instrumento a mesma escala FOIS ${ }^{12}$.
Também é possível verificar aumento do Nível III da escala, indicando que houve aumento do número de crianças com uso de via alternativa de alimentação e que recebem oferta concomitante de alimento por via oral. Tal dado indica que, apesar da intervenção fonoaudiológica poder promover maior eficácia do processo de deglutição, algumas crianças mantém a necessidade do uso da via alternativa de alimentação, não só pelo risco de comprometimento pulmonar, mas também pela necessidade de maior suporte nutricional, uma vez que a alimentação por via oral nestas crianças normalmente demanda maior tempo e compensações, o que pode gerar aumento do gasto enérgico e con- 
sequente necessidade de complemento nutricional por outra via. A má nutrição é um problema comum em crianças com paralisia cerebral ${ }^{13}$.

De acordo com os dados, houve aumento do consumo de dieta pastosa homogênea, e redução da dieta pastosa heterogênea no momento pós-intervenção. Tal dado, juntamente com a verificação de ausência da presença da consistência sólida, sugere uma tendência em indicar uma dieta mais homogênea em pacientes com PC coreoatetoide, com a intenção de promover maior segurança e eficiência na alimentação destas crianças. Além disso, em decorrência da movimentação coreica global nestes pacientes, a mastigação normalmente encontra-se pouco eficaz, aumentando a indicação de uma dieta pastosa homogênea. Pesquisa ${ }^{14}$ afirma que com a instabilidade cervical, movimentos mais finos, como os de língua e de mandíbula, encontram-se com maior comprometimento. Estudo ${ }^{15}$ verifica que texturas mais heterogêneas apresentam controle mais difícil do que texturas mais homogêneas. Em outro estudo ${ }^{16}$ os autores afirmam que após uma intervenção de 10 semanas, a crianças com PC demonstraram otimização do controle de lábios e um padrão de deglutição mais maduro, porém poucas mudanças foram observadas no padrão de mastigação. Afirmam também que a terapia sensório-motora foi efetiva na otimização das habilidades oromotoras, contudo as crianças do estudo dependiam mesmo assim de suplementação calórica, para que ocorresse ganho de peso. Outra pesquisa refere que as alterações da deglutição em crianças com PC consiste na presença de inadequação em fase oral, assim como anormalidades na fase faríngea ${ }^{17}$. As alterações no preparo oral do alimento são frenquentemente associadas à criança com PC ${ }^{18}$.

Com relação à severidade do comprometimento da deglutição houve aumento dos pacientes classificados como apresentando disfagia orofaríngea de grau leve, e diminuição do número daqueles classificados como disfagia orofaríngea grave, no momento pós-intervenção. Assim, como houve redução da ocorrência dos episódios de BCP e de hipersecretividade pulmonar no momento pós-intervenção, também indicando diminuição da gravidade da disfagia em virtude da redução de seus comprometimentos pulmonares. Esta redução da gravidade da disfagia sugere que a intervenção fonoaudiológica, através da manipulação do paciente, mas principalmente através de orientações com relação às consistências alimentares, ao modo de oferta, à postura alimentar, ao utensílio utilizado e ao volume oferecido para cada paciente, colabora para uma alimentação mais segura e eficaz, além de muitas vezes sugerir que a via oral de alimentação não é indicada em certos casos, o que também proporciona melhora na estabilidade clínica e na qualidade de vida do paciente.

Dados da literatura ${ }^{16,19,20}$ verificam que a posição cervical influencia o processo de deglutição, com aumento do risco de aspiração em pacientes com extensão cervical.

A melhora no estado clínico do paciente com PC coreoatetoide também é sugerida pelo aumento na média do peso dos pacientes deste estudo. Cabe dizer que o aumento do peso, que consequentemente proporciona melhora nutricional e clínica, pode ser decorrente da indicação de via alternativa de alimentação pela equipe multidisciplinar em disfagia orofaríngea.

Houve redução dos sinais sugestivos de penetração e/ou aspiração laringotraqueal nos pacientes deste estudo no momento pós-intervenção, assim como os achados de Gisel et al ${ }^{16}$. Este dado também sugere que a intervenção fonoaudiológica contribui para uma deglutição mais eficaz, com menor ocorrência de sinais sugestivos de aspiração traqueal da saliva e/ou alimento. Indica que, mesmo o comprometimento funcional da deglutição permanecendo em maior ou menor grau, as orientações fonoaudiológicas frente aos aspectos da deglutição promovem redução dos riscos de comprometimentos pulmonares, através de compensações que facilitam a proteção das vias aéreas inferiores, como por exemplo, a indicação de uma consistência alimentar mais espessa.

\section{CONCLUSÃO}

A intervenção fonoaudiológica, dentro de uma equipe multidisciplinar em disfagia, em crianças portadoras de PC coreoatetoide promove uma deglutição mais segura e eficaz, com redução dos sinais sugestivos de penetração e/ou aspiração laringotraqueal, dos episódios de BCP e de hipersecretividade pulmonar, e aumento do peso.

\section{AGRADECIMENTOS}

Agradecemos ao Setor de Fonoaudiologia da Associação de Assistência à Criança Deficiente de São Paulo, assim como à equipe multidisciplinar de disfagia desta mesma instituição. 


\section{ABSTRACT}

Purpose: to investigate the efficacy of therapeutic intervention in swallowing and clinical stability of children with choreoathetoid cerebral palsy and oropharyngeal dysphagia. Methods: 11 children with choreoathetoid cerebral palsy, three years and three months old on average, under speech therapy. Data related to staff classification (FOIS scale); dysphagia degree; bronchopneumonia, airway secretions and weight; food consistency; suggestive signs of tracheal aspiration. Data were collected in their medical records before and after therapeutic intervention. Results: data related to FOIS scale indicated that before and after therapeutic intervention, the majority of the patients were in V level, with reduction in the second moment. Increase of the patients in the III and IV levels. After therapeutic intervention, there was reduction of the patients who had liquid foods, increase the use of Jelly-O and homogenic pasty. The severity of dysphagia was reduced as well as the incidence of bronchopneumonia and airway secretions, increase in weight and a reduction of suggestive signs of tracheal aspiration. Conclusion: speech and language therapy intervention, together with the work of a multidisciplinary dysphagia team, promotes better swallowing efficacy and security, with a decrease in suggestive signs of tracheal aspiration, bronchopneumonia and weight increase.

KEYWORDS: Cerebral Palsy; Deglutition Disorders; Speech Therapy

\section{REFERÊNCIAS}

1. Clave P, De Kraa M, Arreola V, Girvent M, Farre $\mathrm{R}$, Palomera $\mathrm{E}$, et al. The effect of bolus viscosity on swallowing function in neurogenic dysphagia. Aliment Pharmacol Ther. 2006; 24(9):1385-94.

2. Padovani AR, Moraes DP, Mangili LD, Andrade CRF. Protocolo fonoaudiológico de avaliação do risco para disfagia (PARD). Rev Soc Bras Fonoaudiol. 2007; 12(3):199-205.

3. Furkim AM, Silva RG. Conceitos e implicações para a prática clínica e para a classificação da disfagia orofaríngea neurogênica. In: Furkim AM, Silva RG. Programas de reabilitação em disfagia neurogênica. São Paulo: Frôntis; 1999. p.1-20.

4. Silva RG. A eficácia da reabilitação em disfagia orofaríngea. Pró-Fono. 2007; 19(1):123-30.

5. Crary MA, Mann GD, Groher ME. Initial psychometric assessment of a functional oral intake scale for dysphagia in stroke patients. Arch Phys Med Rehabil. 2005; 86(8):1516-20.

6. Silva RG, Vieira MM. Disfagia orofaríngea neurogênica em adultos pós acidente vascular encefálico: identificação e classificação. In: Macedo Filho M, Pisani JC, Carneiro J, Gomes G. Disfagia: abordagem multidisciplinar. 3. ed. São Paulo: Frôntis Editorial; 1999. p.29-46.

7. Bobath K. Neurological basis of the treatment of cerebral palsy. 2. ed. London: Heinemann Medical Books; 1980.

8. Rogers B, Arvedson J, Buck G, Smart P, Msall $M$. Characteristics of dysphagia in children with cerebral palsy. Dysphagia. 1994; 9(1):69-73.
9. Furkim AM, Behlau MS, Weckx LLM. Avaliação clínica e videofluoroscópica da deglutição em crianças com paralisia cerebral tetraparética espástica. Arq Neuropsiquiatr. 2003; 61(3A):611-6. 10. Silva AB, Piovesana AMSG, Barcelos IHK, Capellini SA. Evaluación clínica y videofluoroscópica de la deglución en pacientes con parálisis cerebral tetraparésica espástica y atetósica. Rev Neurol. 2006; 42(8):462-5.

11. Rempel G, Moussavi Z. The effect of viscosity on the breath-swallow pattern of young people with cerebral palsy. Dysphagia. 2005; 20(2):108-12.

12. Furkim AM, Sacco ABF. Eficácia da fonoterapia em disfagia neurogênica usando a escala funcional de ingestão por via oral (FOIS) como marcador. Rev CEFAC. 2008; 10(4):503-12. dx.doi.org/10.1590/ S1516-18462008000400010

13. Soylu OB, Unalp A, Uran N, Dizdarer G, Ozgonul FO, Conku A, et al. Effect of nutritional support in children with spastic quadriplegia. Pediatr Neurol. 2008; 39(5):330-4.

14. Seikel JA, King DW, Drumwright DG. Anatomy and physiology for speech, language and hearing. 2.ed. San Diego: Singular; 2000.

15. Gisel EG, Applegate-Ferrante T, Benson JE, Bosma JF. Effect of oral sensorimotor treatment on measures of growth, eating efficiency and aspiration in the dysphagic child with cerebral palsy. Dev Med Child Neurol. 1995; 37(6):528-43.

16. Gisel EG, Applegate-Ferrante T, Benson JE, Bosma JF. Oral-motor skills following sensorimotor therapy in two groups of moderately dysphagic children with cerebral palsy: aspiration vs nonaspiration. Dysphagia. 1996; 11(1):59-71. 
17. Vivone GP, Tavares MMM, Bartolomeu RS, Nemr K. Análise da consistência alimentar e tempo de deglutição em crianças com paralisia cerebral tetraplégica espástica. Rev CEFAC. 2007; 9(4):50411. dx.doi.org/10.1590/S1516-18462007000400011 18. Marrara JL, Duca AP, Dantas RO, Trawitzki LVV, Lima RAC, Pereira JC. Deglutição em crianças com alterações neurológicas: avaliação clínica e videofluoroscópica. Pró-Fono. 2008; 20(4):231-6.
19. Larnert G, Ekberg O. Positioning improves the oral and pharyngeal swallowing function in children with cerebral palsy. Acta Pediatr. 1995; 84(6):689-92.

20. Redstone F, West JF. The importance of postural control for feeding. Pediatr Nurs. 2004; 30(2):97-100.

RECEBIDO EM: 16/03/2009

ACEITO EM: 13/09/2009

Endereço para correspondência:

Carolina Castelli Silvério

Rua Botucatu, 261 ap. 24

São Paulo - SP

CEP: 04023-061

E-mail: carol.silvério@ hotmail.com 